\title{
Mathematical Model of Cytomegalovirus with Vertical Transmision and Mortality Factors
}

\author{
$1^{\text {st }}$ Meli Seprina \\ Mathematics Department \\ State University of Padang \\ Padang, Indonesia \\ meliseprina.meli@gmail.com
}

\author{
$2^{\text {nd }}$ Muhammad Subhan \\ Mathematics Department \\ State University of Padang \\ Padang, Indonesia \\ 13subhan@fmipa.unp.ac.id
}

\author{
$3^{\text {rd }}$ Riry Sriningsih \\ Mathematics Department \\ State University of Padang \\ Padang, Indonesia \\ sriryningsih@yahoo.com
}

\begin{abstract}
Cytomegalovirus (CMV) is a virus belong to herpes family that has incurable infection. The virus attack human immunity which affect miscarriage on pregnant women and fertility of married couples. The goal of this research is to analyze the dynamics of CMV transmission. We make a mathematical model in forms of system of nonlinear differential equations, analyze the factors that cause the transmission, and perform the numerical simulation.
\end{abstract}

Keywords-CMV, Mathematical Model, Dynamical System

\section{INTRODUCTION}

Cytomegalovirus (CMV) infection is one infections in Toxoplasma, Rubella, Cytomegalovirus, and Herpes Simplex virus (TORCH) group [1]. CMV has latent ability inside the organism [2]. CMV infection spreads throughout the world where it is endemic and not depends on season. CMV infection cannot be cured because after infection the virus will stay for lifetime in that person. Therefore, CMV can always reactivate or become active again.

CMV spread can occur vertically or horizontally. Vertical transmission is transmission from a pregnant mother to her fetus [3]. Infections that occur in pregnant women are divided into two, namely primary infection and recurrent infections. Primary infection is an infection that attacks individuals for the first time. Recurrent or reactivation infections are latent infections that become active again [4]. Horizontal transmission is transmission that occurs through sexual intercourse, blood transfusion, tissue transplantation, urine and saliva.

CMV infection can cause systemic infections and attack various kinds of organs including: kidney, pancreas, digestive system, spleen, adrenalin, brain or central nervous system, salivary glands, throat, lungs, liver, and gallbladder. The vertical transmission can cause the fetus to experience developmental disorders (mental retardation), enlarged liver, brain calcification, deafness, and blindness. This infection can cause fetal death in the uterus. The danger of horizontal transmission is that interstitial pneumonia is the main cause of death in bone marrow transplant recipients [5].

The impact of this dangerous CMV requires action to control its spread so that it can be minimized and not spread widely. However, until now, drugs to cure CMV or drugs to kill this virus have not been found. CMV infection depends only on the immune system. Therefore, it is necessary to increase awareness of the danger of CMV to someone who is at high risk of contracting CMV.
Mathematical models are tools that help describe reallife phenomena in the form of mathematical functions or equations in order to obtain a more precise understanding of these real-world problems [6]. This research is expected to provide information about CMV transmission as well as factors that can make CMV infection increase.

\section{METHOD}

This research is basic research using descriptive method. The method used is the analysis of theories relevance with the problems and based on the study of literature. To complete this model, we first study the phenomenon of CMV transmission, and then determine assumptions in the formation of a mathematical model for CMV transmission. After that, we define parameters and variables used in this model, constructing the model, and analyzing and interpreting the behavior of analytical and numerical solution.

\section{RESUlT AND DisCUSSION}

\section{A. CMV transmission model.}

In the formation of the CMV model, we use previous model [7] as a starting point. The population is divided into three groups, namely:

S: Population of individuals susceptible to CMV

E: Population of exposed individuals (infected, but the virus are not active yet).

I: Population of individuals infected with CMV and the virus is already active.

There are several assumptions in the formation of this CMV transmission model, namely:

1. The natural death rate of each compartment is constant and equal.

2. There is no difference between newly infected individuals and those who have long been infected, all individuals who come into contact with infected individuals enter the exposed stage first.

3. Death in exposed population is very rare, so it is assumed that individual death due to $\mathrm{CMV}$ infection only occurs in infected population.

4. Individuals in exposed class become infected at a constant rate of change. 
So that the mathematical model of CMV distribution in the form of a nonlinear differential equation system is obtained as follows:

$$
\begin{aligned}
& \frac{d S}{d t}=\alpha m N-\beta S \frac{I}{N}-\mu S \\
& \frac{d E}{d t}=\alpha(1-m) N+\beta S \frac{I}{N}-(\mu-k) E+f I
\end{aligned}
$$$$
\frac{d I}{d t}=k E-(f+\mu+d) I
$$

The change in the number of individuals from the three compartments is influenced by factors:

\section{$\alpha$ : birth rate}

$\beta$ : rate of CMV transmission due to contact between infectious and susceptible individuals

$\mu:$ natural death rate

$d:$ CMV-related death rate

$k$ : rate of individual change from individual compartment $E$ into compartment $I$

$f: \quad$ rate of individual change from individual compartment $I$ into compartment $E$

$m$ : probability of newborn without CMV infection.

Using all those assumptions, the transmission of CMV can describe like figure 1 .

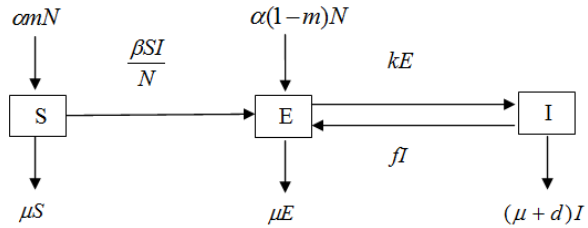

Fig. 1 CMV SEI compartment model.

The system (1) is then scaled with $\mathrm{N}$ to simplify the analysis. The proportion of individuals in each group can be stated as follows:

$$
s=\frac{S}{N}, e=\frac{E}{N}, i=\frac{I}{N}
$$

Since total population is not constant, we use the following derivative rules:

$$
\begin{aligned}
& \frac{d s}{d t}=\frac{1}{N} \frac{d S}{d t}-\frac{s}{N} \frac{d N}{d t} \\
& \frac{d e}{d t}=\frac{1}{N} \frac{d E}{d t}-\frac{e}{N} \frac{d N}{d t} \\
& \frac{d i}{d t}=\frac{1}{N} \frac{d I}{d t}-\frac{i}{N} \frac{d N}{d t}
\end{aligned}
$$

to get the new proportion system of equations:

$$
\frac{d s}{d t}=\alpha m-\beta s i-\alpha s+s d i
$$

$$
\begin{array}{r}
\widehat{s}^{*}=\frac{1}{(d-\beta) k} \sqrt{\left(\begin{array}{l}
\left(d^{2}-d \beta\right)+\left(-d^{2}-d f-d k-2 d \alpha+d \beta+f \beta+k \beta+\alpha \beta\right) \\
\left.+\left(d k+d \alpha+f \alpha+k \alpha-k \beta+\alpha^{2}\right)+\alpha m k-\alpha k\right)
\end{array}\right) d} \\
-\frac{\alpha d}{(d-\beta) k}-\frac{\alpha f}{(d-\beta) k}-\frac{\alpha m}{(d-\beta) \sqrt{\left(\begin{array}{l}
\left.\left(d^{2}-d \beta\right)+\left(-d^{2}-d f-d k-2 d \alpha+d \beta+f \beta+k \beta+\alpha \beta\right)\right) \\
\left.+\left(d k+d \alpha+f \alpha+k \alpha-k \beta+\alpha^{2}\right)+\alpha m k-\alpha k\right)
\end{array}\right)}} \\
-\frac{\alpha}{(d-\beta)}-\frac{\alpha^{2}}{(d-\beta) k}+\frac{\alpha}{(d-\beta) \sqrt{\left(\begin{array}{l}
\left.\left(d^{2}-d \beta\right)+\left(-d^{2}-d f-d k-2 d \alpha+d \beta+f \beta+k \beta+\alpha \beta\right)\right) \\
\left.+\left(d k+d \alpha+f \alpha+k \alpha-k \beta+\alpha^{2}\right)+\alpha m k-\alpha k\right)
\end{array}\right)}}
\end{array}
$$

$$
\begin{aligned}
& \frac{d e}{d t}=\beta s i+\alpha-\alpha m-k e+f i-\alpha e+e d i \\
& \frac{d i}{d t}=k e-f i-d i-\alpha i+d i^{2}
\end{aligned}
$$

System of equations (5) is a nonlinear system resulted from the transformation of the SEI model (1).

\section{B. Basic Reproduction Ratio}

The basic reproduction ratio $R_{0}$ can be determined using the formula [8]:

where is

$$
K=F V^{-1}
$$

$$
F=\left[\begin{array}{cc}
-k-\alpha+\delta i & \beta s+\gamma+e \delta \\
k & -\gamma-\delta-\alpha+2 \delta i
\end{array}\right]
$$

Substituting $E_{0}(\widehat{s}, \hat{e}, \hat{i})=(1,0,0)$ into matrix $F$,

$$
F=\left[\begin{array}{cc}
-k-\alpha & \beta+f \\
k & -f-d
\end{array}\right] \text { dan } V=\left[\begin{array}{cc}
\mu+k & 0 \\
0 & f+\mu+d
\end{array}\right]
$$

we get

$$
\begin{aligned}
& R_{0}=\frac{-\left(\frac{k+\alpha}{\mu+k}+\frac{d+f)}{f+\mu+d}\right)}{2} \\
& +\frac{\sqrt{\left(\frac{k+\alpha}{\mu+k}+\frac{d+f)}{f+\mu+d}\right)^{2}-4\left[\left(\frac{k+\alpha}{\mu+k}\right)\left(\frac{d+f)}{f+\mu+d}\right)-\left(\frac{k}{\mu+k}\right)\left(\frac{\beta+\alpha}{f+\mu+d}\right)\right]}}{2}
\end{aligned}
$$

\section{Qualitative Analysis}

To understand the long-term behavior, we want to describe the dynamical behavior of the model by finding the fixed points and their stability [9]. We get two fixed points of the model, namely virus free and endemic fixed point.

\section{1) Virus-free point}

This point represents a condition where there is no more transmission of infection in the population, or when $i=0$.

$$
\left(\widehat{s}^{*}, \widehat{e}^{*}, \hat{i}^{*}\right)=(1,0,0)
$$

\section{2) Endemic point}

This point represents condition where there always be transmission of infection in the population, or when $i \neq 0$. 


$$
\begin{aligned}
\hat{e}^{*}=- & \frac{1}{k}\left(\sqrt{\left(\begin{array}{l}
\left(d^{2}-d \beta\right)+\left(-d^{2}-d f-d k-2 d \alpha+d \beta+f \beta+k \beta+\alpha \beta\right) \\
\left.+\left(d k+d \alpha+f \alpha+k \alpha-k \beta+\alpha^{2}\right)+\alpha m k-\alpha k\right)
\end{array}\right)^{2} d}\right) \\
+ & \frac{1}{k}\left(\sqrt{\left(\begin{array}{l}
\left(d^{2}-d \beta\right)+\left(-d^{2}-d f-d k-2 d \alpha+d \beta+f \beta+k \beta+\alpha \beta\right) \\
\left.+\left(d k+d \alpha+f \alpha+k \alpha-k \beta+\alpha^{2}\right)+\alpha m k-\alpha k\right)
\end{array}\right) d}\right) \\
& +\frac{1}{k}\left(\sqrt{\left(\begin{array}{l}
\left(d^{2}-d \beta\right)+\left(-d^{2}-d f-d k-2 d \alpha+d \beta+f \beta+k \beta+\alpha \beta\right) \\
\left.+\left(d k+d \alpha+f \alpha+k \alpha-k \beta+\alpha^{2}\right)+\alpha m k-\alpha k\right)
\end{array}\right) f}\right) \\
& +\frac{1}{k}\left(\sqrt{\left(\begin{array}{l}
\left(d^{2}-d \beta\right)+\left(-d^{2}-d f-d k-2 d \alpha+d \beta+f \beta+k \beta+\alpha \beta\right) \\
\left.+\left(d k+d \alpha+f \alpha+k \alpha-k \beta+\alpha^{2}\right)+\alpha m k-\alpha k\right)
\end{array}\right) \alpha}\right)
\end{aligned}
$$

$\hat{i}^{*}=\sqrt{\left(\begin{array}{l}\left(d^{2}-d \beta\right)-Z^{3}+\left(-d^{2}-d f-d k-2 d \alpha+d \beta+f \beta+k \beta+\alpha \beta\right) \\ \left.+\left(d k+d \alpha+f \alpha+k \alpha-k \beta+\alpha^{2}\right)+\alpha m k-\alpha k\right)\end{array}\right)}(6)$

Using these equations:

$\frac{d s}{d t}=f_{1}, \frac{d e}{d t}=f_{2}, \frac{d i}{d t}=f_{3}$

where

$$
\begin{gathered}
f_{1}(s, e, i)=\alpha m-\beta s i-\alpha s+s d i \\
f_{2}(s, e, i)=\beta s i+\alpha-\alpha m-k e+f i-\alpha e+e d i \\
f_{3}(s, e, i)=k e-f i-d i-\alpha i+d i^{2}
\end{gathered}
$$

we get the Jacobian matrix for the system is

$$
J(f(E))=\left|\begin{array}{ccc}
-(\beta i+\alpha-d i) & 0 & -\beta s+s d \\
\beta i & -(k+\alpha-d i) & \beta s+f+e d \\
0 & k & -(f+d+\alpha-2 d i)
\end{array}\right|
$$

For virus-free point $E_{0}=(1,0,0)$, the Jacobian is

$$
J\left(f\left(E_{0}\right)\right)=\left|\begin{array}{ccc}
-\alpha & 0 & -\beta+d \\
0 & -(k+\alpha) & \beta+f \\
0 & k & -(f+d+\alpha)
\end{array}\right|
$$

so its characteristic equation is

$$
(\lambda+\alpha)\left[\lambda^{2}+(d+f+k+2 \alpha) \lambda+k d+\alpha d+\alpha f+k \alpha-k \beta+\alpha^{2}\right]=0
$$

The eigenvalues for this Jacobian are $\lambda_{1}=-\alpha$ and from the second term that we can write as $\lambda^{2}+a_{1} \lambda+a_{2}=0$

where $a_{1}=d+f+k+2 \alpha$

$$
a_{2}=k d+\alpha d+\alpha f+k \alpha-k \beta+\alpha^{2}
$$

we have the eigenvalues

$\lambda_{2}=\frac{-a_{1}-\sqrt{a_{1}^{2}-4 a^{2}}}{2} \quad \lambda_{3}=\frac{-a_{1}+\sqrt{a_{1}^{2}-4 a^{2}}}{2}$

When $R_{0}<1$, then both $\lambda_{2}$ and $\lambda_{3}$ have negative real part, but when $R_{0}>1, \lambda_{3}$ has positive real part. We can conclude that $E_{0}$ is a stable when there is no epidemic and unstable when there is epidemic.
The Jacobian for $E_{1}=\left(\hat{s}^{*}, \hat{e}^{*}, \hat{i}^{*}\right)$ is:

$$
J\left(f\left(E_{1}\right)\right)=\left|\begin{array}{ccc}
-\left(\beta \hat{i}^{*}+\alpha-d \hat{i}^{*}\right) & 0 & -\beta \hat{s}^{*}+\hat{s}^{*} d \\
\beta \hat{i}^{*} & -\left(k+\alpha-d \hat{i}^{*}\right) & \beta \hat{s}^{*}+f+\hat{e}^{*} d \\
0 & k & -\left(f+d+\alpha-2 d \hat{i}^{*}\right)
\end{array}\right|
$$

and its characteristic equation is

$\lambda+\left(\beta \hat{i}^{*}+\alpha-d \hat{i}^{*}\right)\left[\left(\lambda+\left(k+\alpha-d \hat{i}^{*}\right)\right)\left(\lambda+\left(f+d+\alpha-2 d \hat{i}^{*}\right)\right)\right]$ $-\beta \hat{i}^{*}\left[-k\left(\beta \hat{s}^{*}-\hat{s}^{*} d\right)\right]=0$

The analytical analysis for this point isn't succesful yet, so we use the numerical approach to achieve some conclussion.

\section{Numerical Simulation}

We use two set of parameters for this numerical simulation:

1) SetA:

$\alpha=0.000051, \mu=0.000039, d=0.000001, k=0.0000035$

$\beta=0.000003, m=1, f=0.00000001$

which give us $R_{0}=0.438$

2) $\operatorname{SetB}$ :

$\alpha=0.000051, \mu=0.000039, d=0.000021, k=0.000508$

$\beta=0.00081, m=0.21, f=0.000029$

which give $R_{0}=2.068$ and endemic point $E_{1}=(0.0149,0.1387,0.8464)$

Using these initial values:

$$
\begin{aligned}
& s(0)=1, e(0)=0, i(0)=0 \\
& s(0)=0.99, e(0)=0.0818, i(0)=0.00882 \\
& s(0)=0.98, e(0)=0.0014, i(0)=0.0186
\end{aligned}
$$

The dynamical behavior of the systems showed in Figure 2 confirm that the virus-free point is stable.

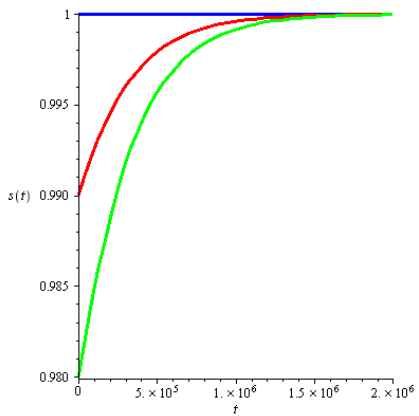

Trajectory of $\mathrm{s}(\mathrm{t})$

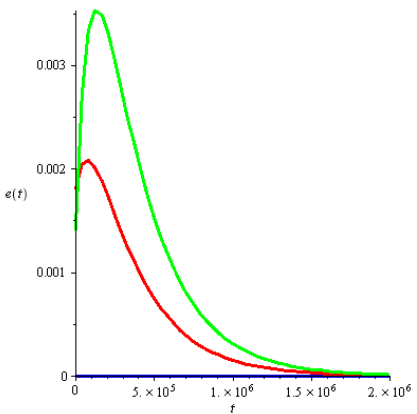

Trajectory of e(t)

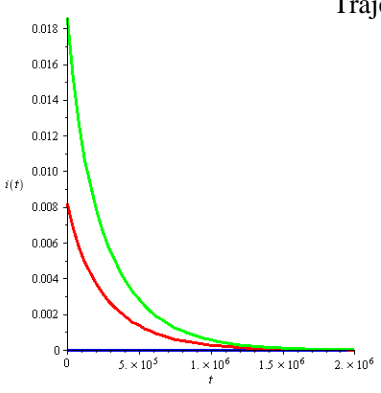

Trajectory of $\mathrm{i}(\mathrm{t})$

Fig. 2. Trajectories of system when $R_{0}<1$. 
Figure 3 shows that the virus-free point is unstable and endemic point is stable. For this simulation we use initial values:

$$
\begin{aligned}
& s(0)=0.0149, e(0)=0.1387, i(0)=0.8464 \\
& s(0)=1, e(0)=0, i(0)=0 \\
& s(0)=0.5, e(0)=0.3, i(0)=0.2
\end{aligned}
$$

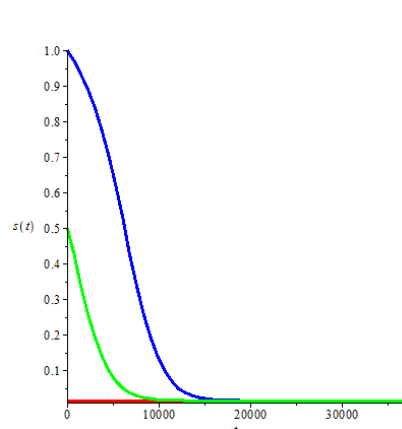

Trajectory of $\mathrm{s}(\mathrm{t})$

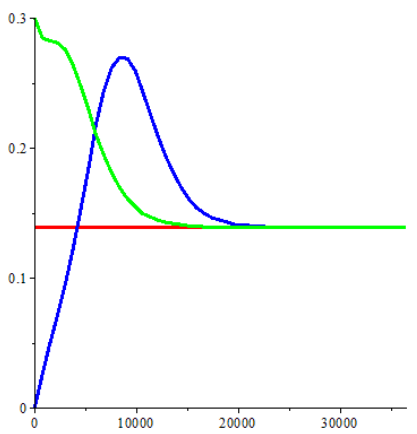

Trajectory of $\mathrm{t}(\mathrm{t})$

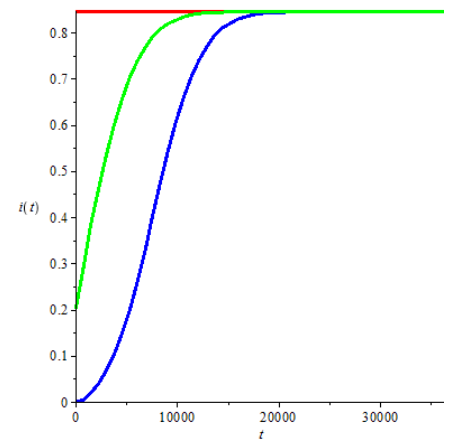

Trajectory of i(t)

Fig. 3. Trajectories of system when $R_{0}>1$

\section{CONCLUSION}

Based on qualitative analysis and numerical simulation, it can be concluded that the mathematical model of CMV with vertical transmission and mortality factors is in form of SEI model has two fixed point which theirs stability depends on the basic reproduction ratio. The factors that dominantly affect the increasing number of CMVinfected individuals are rate of CMV transmission due to contact between infected individuals and susceptible individuals and rate of change from exposed to infected compartment.

\section{REFERENCES}

[1] G. A. M, "Prevalensi Seropositif IgM/IgG Cytomegalovirus pada Populasi Wanita Pra-nikah dengan Riwayat Konsumsi Makan Lesehan". Jurnal Mutiara Medika.Yogyakarta. Indonesia, vol. 12, pp. 124-131, Mei 2012.

[2] Soedarto, "Virologi Klinik". Jakarta: Sagung Seto. 2010.

[3] J. Meier, et al. "Human cytomegalovirus reactivation during lactation and mother-to-child transmission in preterm infants." Journal of clinical microbiology, vol 43, 1318-1324, 2005

[4] R. F Pass., and Brenna Anderson. "Mother-to-child transmission of cytomegalovirus and prevention of congenital infection". Journal of the Pediatric Infectious Diseases Society, vol 3, 2-6, 2014
[5] P. Ljungman, Paul Griffiths, and Carlos Paya. "Definitions of cytomegalovirus infection and disease in transplant recipients". Clinical infectious diseases, vol 34, 1094-1097, 2002

[6] Widiarti and Sutimin, "Buku Ajar Pemodelan Matematika". Semarang: Universitas Diponegoro. 2007.

[7] R. Sriningsih, M. Subhan, and M. L. Nasution "Mathematical Model of Cytomegalovirus (CMV) Disease". IOP Conference Series vol.335 Issue 1 pp 012050, 2018.

[8] M. Martcheva, "An Introduction to Mathematical Epidemiology". New York: Spinger, 2015

[9] J. W Cain., dan Reynold. "Ordinary and Partial Differential Equation: An Introduction to Dynamical Systemd". Virginia: Commonwealth University. 2010. 